Pacific Journal of Mathematics

SOME AFFINE GEOMETRIC ASPECTS OF OPERATOR 


\title{
SOME AFFINE GEOMETRIC ASPECTS OF OPERATOR ALGEBRAS
}

\author{
YAAKov FRIEDMAN AND BERNARd RusSO \\ Dedicated to the memory of Henry Abel Dye
}

\begin{abstract}
The predual of a von Neumann algebra is shown to be a neutral strongly facially symmetric space, thereby suggesting an affine geometric approach to operator algebras and their non-associative analogues. Geometric proofs are obtained for the polar decompositions of normal functionals in ordered and non-ordered settings.
\end{abstract}

A fundamental problem in the operator algebraic approach to quantum mechanics is to determine those algebraic structures in Banach spaces which are characterized by a set of geometrical axioms defining the quantum mechanical measuring process. This problem was solved in the context of ordered Banach spaces by Alfsen, Hanche-Olsen, and Shultz ([2], [1]) and led to a characterization of the state spaces of $J B^{*}$-algebras and $C^{*}$-algebras. The main thrust of the present authors' recent research has been to find those algebraic structures induced on (unordered) Banach spaces in which such quantum mechanical axioms are satisfied. This project, which was initiated in [14] and [15] using the affine geometry of the dual unit ball, is used here to give a geometric proof of the Tomita-Sakai-Effros polar decomposition of a normal functional on a von Neumann algebra.

Thus, the purpose of this partially expository paper is to show the richness and power of the affine geometric structure of the dual space of an operator algebra, by working in a purely geometric model. Indeed, since this geometry can be described in terms of the underlying real structure, it can be used to obtain new results in the real structure of operator algebras and in the structure of real operator algebras. For example, by using this approach, Dang ([5]) has shown that a real-linear isometry of a $C^{*}$-algebra is the sum of a linear and a conjugate linear isometry, and hence is multiplicative, thereby obtaining a real analogue of Kadison's non-commutative extension of the BanachStone Theorem.

The category of strongly facially symmetric (SFS) spaces (simply called facially symmetric spaces in [14] and [15]) has been shown to 
be an appropriate vehicle for the study of this problem. Strongly facially symmetric spaces include the preduals of von Neumann algebras (cf. $\S 2$ below), and more generally of $J B W^{*}$-triples (cf. $\S 3$ below), and can therefore serve as a geometric order-free model in which to study operator algebras and their non-associative analogues. This project may also be viewed as an attempt to place the characterizations of von Neumann algebras and $J B W$-algebras in terms of self-dual homogeneous cones ([4], [19]) in a context which is free of a global order structure.

A discrete spectral theorem for an arbitrary element in the dual of a reflexive SFS space was obtained in [14] by using the basic notions of orthogonality, projective unit, norm exposed face, symmetric face, generalized tripotent, and generalized Peirce decomposition, which were introduced and developed in this purely geometric setting. Moreover, a one-to-one correspondence was established between generalized tripotents and norm exposed faces, analogous to the correspondence between projections in a von Neumann algebra and norm closed faces in its normal state space (cf. [9]). This constitutes an important link between geometry and algebra in this context.

We next discuss the notion of compatibility. It is often useful to decompose a space simultaneously with respect to a family of individual decompositions. It is therefore important to know conditions under which this joint decomposition does not depend on the order, i.e. when the corresponding projections all commute. In this case we say that the members of the family are compatible.

In the globally ordered case, faces correspond to idempotents, and compatibility implies commutativity of the idempotents. Therefore, it is not possible to study non-commutative phenomena in this framework without abandoning compatibility, and thus increasing significantly the complexity of the problem. On the other hand, by dropping the requirement of a global order structure, it has been possible to describe non-commutative objects by using compatible families. This is illustrated by the proof of the classification of $J B W^{*}$-triple factors of type $I$ in [6].

In $\S 1$ we review the basic affine geometric notions needed in the proof of the geometric polar decomposition ([15, Theorem 4.3]), and give necessary and sufficient conditions for compatibility (Theorem 1.2), considerably sharpening one of the main results of [15]. In $\S 2$ we develop enough elementary machinery of operator algebras in order to show that the predual of a von Neumann algebra is a neutral strongly 
facially symmetric space (Theorem 2.11). It is then a simple matter to combine Theorems 1.3 and 2.11 to obtain a geometric proof of the Tomita-Sakai-Effros polar decomposition. A generalization of the results of $\S 2$ appears in $\S 3$, which introduces the $J B^{*}$-triples.

1. Compatibility in weakly facially symmetric spaces. In this section we shall give several necessary and sufficient conditions for compatibility of elements in a purely geometric setting. A consequence of this result constitutes one step in the proof of the geometric polar decomposition ([15, Theorem 4.3]), which we state here. All other definitions from [14] and [15] which are needed for our proof of compatibility will be recalled here for the convenience of the reader.

We shall say that elements $f$ and $g$ of a normed space $Z$ are $o r$ thogonal if they satisfy

$$
\|g+f\|=\|g-f\|=\|g\|+\|f\|,
$$

or if one of them is zero (cf. $[14, \S 1]$ or $[15, \S 1]$. We shall write $f \diamond g$ to indicate that $f$ and $g$ are orthogonal.

Let $K$ be a convex set. A face of $K$ is a non-empty convex subset $F$ of $K$ with the following property: if $f \in F$ and $g, h \in K$ satisfy $f=\lambda g+(1-\lambda) h$ for some $\lambda \in(0,1)$, then $g, h \in F$. An important example is: $K=Z_{1}=$ the unit ball of $Z$ and $F=\{f \in K: f(x)=1\}$ for some element $x \in Z^{*}$ of norm 1 . We shall denote this set $F$ which is either empty or a face (called a norm exposed face), by $F_{x}$. Note that $F_{x} \cap F_{y}=F_{z}$ where $z=(x+y) / 2$. The following is immediate from the definitions.

REMARK 1.1. Let $T$ be a linear isometry of a normed space $Z$ onto itself. For each norm exposed face $F_{x}$ in $Z_{1}$,

$$
T^{-1}\left(F_{x}\right)=F_{T^{*} x}
$$

We say that $Z$ is facially linearly complemented if it is a real or complex normed space in which the orthogonal complement

$$
F^{\diamond}=\{g \in Z: g \diamond f, \forall f \in F\}
$$

of every norm exposed face $F$ is a linear subspace. An element $u \in Z^{*}$ is called a projective unit if $\|u\|=1$ and $\left\langle u, F_{u}^{\diamond}\right\rangle=0$. Let $\mathscr{F}$ and $\mathscr{U}$ denote the collections of norm exposed faces of $Z_{1}$ and projective units in $Z^{*}$, respectively. The map

$$
\mathscr{U} \ni u \mapsto F_{u} \in \mathscr{F}
$$


is not onto in general ([14, p. 269]). In order to obtain the bijection between distinguished subsets of $\mathscr{F}$ and $\mathscr{U}$, we need to recall the definitions of symmetric face and generalized tripotent.

Motivated by measuring processes in quantum mechanics, we define a symmetric face to be a norm exposed face $F$ in $Z_{1}$ with the following property: there is a linear isometry $S_{F}$ of $Z$ onto $Z$, with $S_{F}^{2}=I$, such that the fixed point set of $S_{F}$ is $(\overline{\mathrm{sp}} F) \oplus F^{\diamond}$ (topological direct sum). In particular, $F^{\diamond}$ is a closed linear space.

For each symmetric face $F$ we define contractive projections $P_{k}(F)$, $k=0,1,2$ on $Z$ as follows. First $P_{1}(F)=\frac{1}{2}\left(I-S_{F}\right)$ is the projection on the -1 eigenspace of $S_{F}$. Next we define $P_{2}(F)$ and $P_{0}(F)$ as the projections of $Z$ onto $\overline{\mathrm{sp}} F$ and $F^{\diamond}$ respectively, so that $P_{2}(F)+$ $P_{0}(F)=\frac{1}{2}\left(I+S_{F}\right)$ is the projection on the +1 eigenspace of $S_{F}$. These projections are called generalized Peirce projections. Note that

$$
P_{2}(F)+P_{1}(F)+P_{0}(F)=I \text { and } S_{F}=P_{2}(F)-P_{1}(F)+P_{0}(F) \text {. }
$$

A real or complex normed space $Z$ is said to be weakly facially symmetric (WFS) if every norm exposed face in $Z_{1}$ is symmetric. Hence a WFS space is facially linearly complemented.

A generalized tripotent is a projective unit $u \in \mathscr{U}$ with the property that $F_{u}$ is a symmetric face and $S_{F u}^{*} u=u$. The importance of this concept is explained by the following. Denote by $\mathscr{G} \mathscr{T}$ and $\mathscr{S F}$ the collections of generalized tripotents and symmetric faces respectively. According to [15, Proposition 1.6], for any WFS space $Z$, the map

$$
\mathscr{G T} \ni u \mapsto F_{u} \in \mathscr{S F}
$$

is a bijection of the set of generalized tripotents and the set of symmetric faces.

We now define the orthogonality of generalized tripotents and other elements of the dual space $Z^{*}$ of a WFS space $Z$. Elements $a, b \in Z^{*}$, $Z$ any normed space, are orthogonal ${ }^{1}$ if there is a symmetric face $F \subset Z_{1}$ such that either
1. $a \in P_{2}(F)^{*}\left(Z^{*}\right)$ and $b \in P_{0}(F)^{*}\left(Z^{*}\right)$; or
2. $a \in P_{0}(F)^{*}\left(Z^{*}\right)$ and $b \in P_{2}(F)^{*}\left(Z^{*}\right)$.

We shall write $a \diamond b$ or $b \diamond a$ to indicate this relation.

We next recall the notion of neutrality. A contractive projection $Q$ on a normed space $X$ is said to be neutral if for each $\xi \in X$, $\|Q \xi\|=\|\xi\|$ implies $Q \xi=\xi$. A normed space $Z$ is neutral if for every

${ }^{1}$ Later we shall use the term orthogonal in connection with elements of a $C^{*}$-algebra. By Theorem 2.11, the two definitions are consistent. 
symmetric face $F$, the projection $P_{2}(F)$ corresponding to some choice of symmetry $S_{F}$, is neutral. An interesting property of neutrality is that in a neutral WFS space, a symmetry $S_{F}$ as well as the generalized Peirce projections, are uniquely determined by the norm exposed face $F$ ([15, Theorem 2.4]).

We next define the notion of compatibility and prove the important characterization theorem. For an analogous result in a purely algebraic setting, see [23].

Two generalized tripotents $u$ and $v$ are said to be compatible if their generalized Peirce projections commute, i.e.

$$
\left[P_{k}\left(F_{u}\right), P_{j}\left(F_{v}\right)\right]=0 \text { for } k, j \in\{0,1,2\} .
$$

The following theorem gives a characterization of compatibility which sharpens a previous result of the authors. For convenience, we adopt the following notations, where $u$ is a generalized tripotent in the dual of a WFS space $Z: P_{k}(u)=P_{k}\left(F_{u}\right), k=0,1,2 ; U=Z^{*}$, $Z_{k}(u)=P_{k}(u)(Z) ; U_{k}(u)=P_{k}(u)^{*}(U) ; S_{u}=S_{F_{u}}$. Also, the inverse of the map (1) will be denoted by $F \mapsto v_{F}$.

THEOREM 1.2. Let $Z$ be a neutral WFS space, and let $u, v \in \mathscr{G T}$. The following are equivalent.

(1) $u$ and $v$ are compatible;

(2) $S_{v}^{*} u \in U_{2}(u)$

(2') $S_{u}^{*} v \in U_{2}(v)$

(3) $u=u_{2}+u_{1}+u_{0}$ with $u_{j} \in U_{j}(v) \cap U_{2}(u), j \in\{0,1,2\}$;

$\left(3^{\prime}\right) v=v_{2}+v_{1}+v_{0}$ with $v_{j} \in U_{j}(u) \cap U_{2}(v), j \in\{0,1,2\}$.

Proof.

(1) $\Rightarrow$ (3): $P_{i}(v)^{*} u=P_{i}(v)^{*} P_{2}(u)^{*} u=P_{2}(u)^{*} P_{i}(v)^{*} u \in U_{i}(v) \cap$ $U_{2}(u)$.

(3) $\Rightarrow(2): S_{v}^{*} u=u_{2}-u_{1}+u_{0} \in U_{2}(u)$.

$(2) \Rightarrow(1)$ : By Remark 1.1, $S_{v}\left(F_{u}\right)=F_{S_{v}^{*} u}$, and since $S_{v}^{*} u \in U_{2}(u)$ we have by [15, Theorem 2.3] that $S_{v}\left(F_{u}\right) \subset Z_{2}(u)$. Thus

$$
P_{2}(u) S_{v} P_{2}(u)=S_{v} P_{2}(u) .
$$

Since $S_{v}^{2}=I, S_{v}\left(Z_{2}(u)\right)=Z_{2}(u)$. Since by [15, Proposition 1.5], $Z_{2}(u) \diamond Z_{0}(u)$, we have $Z_{0}(u) \subset Z_{2}(u)^{\diamond}$. But $F_{u} \subset Z_{2}(u)$ implies $Z_{0}(u)=F_{u}^{\diamond} \supset Z_{2}(u)^{\diamond}$ so $Z_{0}(u)=Z_{2}(u)^{\diamond}$. Therefore

$$
S_{v}\left(Z_{0}(u)\right)=S_{v}\left(Z_{2}(u)^{\diamond}\right)=S_{v}\left(Z_{2}(u)\right)^{\diamond}=Z_{2}(u)^{\diamond}=Z_{0}(u),
$$


from which we get

$$
P_{0}(u) S_{v} P_{0}(u)=S_{v} P_{0}(u) .
$$

For $S_{v}^{*}$, by [15, Theorem 2.3] and Remark 1.1, we obtain

$$
\begin{aligned}
S_{v}^{*}\left(U_{k}(u)\right) & =\overline{\mathbf{s p}}^{w^{*}}\left\{S_{v}^{*}\left(v_{H}\right): H \in \mathscr{S F}, H \subset Z_{k}(u)\right\}, \\
& =\overline{\mathbf{s p}}^{w^{*}}\left\{v_{S_{v}(H)}: H \in \mathscr{S F}, H \subset Z_{k}(u)\right\},
\end{aligned}
$$

for $k=0$ or 2. Since (2) and (3) imply that $S_{v}(H) \subset Z_{k}(u)$, we have

$$
P_{2}(u)^{*} S_{v}^{*} P_{2}(u)^{*}=S_{v}^{*} P_{2}(u)^{*},
$$

and

$$
P_{0}(u)^{*} S_{v}^{*} P_{0}(u)^{*}=S_{v}^{*} P_{0}(u)^{*} .
$$

Using (4) and (2) we have

$$
\begin{aligned}
P_{2}(u)^{* *} S_{v}^{* *} & =P_{2}(u)^{* *} S_{v}^{* *} P_{2}(u)^{* *} \\
& =\left(P_{2}(u) S_{v} P_{2}(u)\right)^{* *} \\
& =\left(S_{v} P_{2}(u)\right)^{* *}=S_{v}^{* *} P_{2}(u)^{* *}
\end{aligned}
$$

Thus $\left[P_{2}(u), S_{v}\right]=0$.

Similarly, (3) and (5) imply $\left[P_{0}(u), S_{v}\right]=0$. Since $P_{1}(u)=I-$ $P_{2}(u)-P_{0}(u)$ and $P_{1}(v)=\left(I-S_{v}\right) / 2$, we have

$$
\left[P_{j}(u), S_{v}\right]=0=\left[P_{j}(u), P_{1}(v)\right] \text { for } j \in\{0,1,2\} .
$$

Since $P_{2}(v)+P_{0}(v)=\left(I+S_{v}\right) / 2$, (6) implies

$$
\left(P_{2}(v)+P_{0}(v)\right)\left(Z_{j}(u)\right) \subset Z_{j}(u), \quad \text { for } j \in\{0,1,2\} .
$$

Using the fact that $Z_{2}(v) \diamond Z_{0}(v)$, from [15, Remark 1.3] (for $j=0$ ) and [15, Remark 3.2] (for $j=2$ ) we have

$$
P_{i}(v)\left(Z_{j}(u)\right) \subset Z_{j}(u) \text { for } i, j \in\{0,2\} \text {, }
$$

i.e.

$$
P_{i}(v) P_{j}(u)=P_{j}(u) P_{i}(v) P_{j}(u), \quad \text { for } i, j \in\{0,2\} .
$$

Moreover $Q:=P_{i}(v) P_{j}(u)$ is a neutral contractive projection with range $Z_{i}(v) \cap Z_{j}(u)$, for $i, j \in\{0,2\}$.

Obviously, the subspace $Q^{*}(Z)$ contains $U_{i}(v) \cap U_{j}(u)$. If these spaces are not equal, we may choose a $\varphi \in Q(Z)$ such that $\|\varphi\|=1$ and

$$
\varphi\left(U_{i}(v) \cap U_{j}(u)\right)=0
$$


Then there exists $x \in Q^{*}(U),\|x\|=1$ with $\varphi \in F_{x}$. By neutrality of $Q, F_{x} \subset Q(Z)$ and by [15, Theorem 2.3], $v_{F_{x}} \in U_{i}(v) \cap U_{j}(u)$. Hence $\varphi\left(v_{F_{x}}\right)=0$, contradicting $\varphi \in F_{x}$. Thus $Q^{*}(U)=U_{i}(v) \cap U_{j}(u)$.

Now let $R:=P_{i}(v) P_{j}(u) P_{i}(v)$. From (8) it follows that

$$
R^{2}=P_{i}(v) P_{j}(u) P_{i}(v) P_{j}(u) P_{i}(v)=R
$$

so that $R$ is a neutral contractive projection. For any $\varphi \in Z$ we have

$$
\|R \varphi\|=\left\|R^{2} \varphi\right\|=\left\|P_{i}(v) P_{j}(u) R \varphi\right\| \leq\left\|P_{j}(u) R \varphi\right\| \leq\|R \varphi\| .
$$

Since $P_{j}(u)$ is neutral for $j=0$ or $2, R \varphi=P_{j}(u) R \varphi$. Thus $R(Z)=$ $Z_{j}(u) \cap Z_{i}(v)$. This implies, as above, that $R^{*}(U)=U_{i}(v) \cap U_{j}(u)$ and thus by [15, Lemma 2.2], $Q=R$, i.e.

$$
P_{i}(v) P_{j}(u)=P_{i}(v) P_{j}(u) P_{i}(v), \quad \text { for } i, j \in\{0,2\} .
$$

From this it follows that (for $i \in\{0,2\}$ )

$$
\begin{aligned}
P_{i}(v) P_{1}(u) & =P_{i}(v)\left[P_{2}(u)+P_{1}(u)+P_{0}(u)\right] P_{i}(v) P_{1}(u) \\
& =\left(P_{i}(v) P_{1}(u)\right)^{2},
\end{aligned}
$$

implying, by neutrality of $P_{i}(v)$,

$$
P_{i}(v) P_{1}(u)=P_{1}(u) P_{i}(v) P_{1}(u), \quad \text { for } i \in\{0,2\} .
$$

Finally, using (8) and (10), we have, for $i, j \in\{0,2\}$,

$$
\begin{aligned}
P_{j}(u) P_{i}(v) & =P_{j}(u) P_{i}(v)\left[P_{2}(u)+P_{1}(u)+P_{0}(u)\right] \\
& =P_{j}(u) P_{i}(v) P_{j}(u) \\
& =P_{i}(v) P_{j}(u)
\end{aligned}
$$

i.e., $\left[P_{i}(v), P_{j}(u)\right]=0$ for $i, j \in\{0,2\}$. This fact, together with (6) shows that $u$ and $v$ are compatible.

By symmetry of $(1),\left(2^{\prime}\right)$ and $\left(3^{\prime}\right)$ are equivalent to (1).

As a consequence of Theorem 1.2 we obtain [15, Theorem 3.3], one of the main results of [15].

A WFS space $Z$ is strongly facially symmetric (SFS) if for every norm exposed face $F$, and every $y \in Z^{*}$ with $\|y\|=1$ and $F \subset F_{y}$, we have $S_{F}^{*} y=y$, where $S_{F}$ denotes a symmetry corresponding to $F$. In a SFS space, the generalized tripotents coincide with the projective units.

Another main result of [15] is the following, which is a geometric polar decomposition. 
THEOREM 1.3 (Theorem 4.3 of [15]). Let $Z$ be a neutral SFS space. For any $f \in Z$ with $\|f\|=1$, there is a unique generalized tripotent $v$ such that

(a) $f \in F_{v}$;

(b) $\left\langle v,\{f\}^{\circ}\right\rangle=0$.

Moreover,

(c) $F_{v}$ is the smallest norm exposed face containing $f$;

(d) $f$ is faithful in the sense that

$$
f(u)=\left\|P_{2}(u) f\right\|>0 \quad \text { for any } u \in \mathscr{G g} \text { with } F_{u} \subset F_{v} .
$$

2. Application to operator algebras. In this section we will show that the predual of a von Neumann algebra is a neutral strongly facially symmetric space, and that the set of generalized tripotents coincides with the set of partial isometries in the von Neumann algebra. These facts will be used to give a geometric proof of the Tomita-Sakai-Effros polar decomposition of a normal functional.

If $v$ is a partial isometry in a $C^{*}$-algebra $A$, then with $l=v v^{*}$ and $r=v^{*} v$, projections $E(v), F(v)$, and $G(v)$ on $A$ are defined by

$$
\left.\begin{array}{l}
E(v) x=l x r \\
F(v) x=(1-l) x(1-r) ; \\
G(v) x=l x(1-r)+(1-l) x r
\end{array}\right\}, \quad \text { for } x \in A,
$$

and yield the familiar "matrix" representation of $x$ :

$$
x \sim\left[\begin{array}{cc}
E(v) x & l x(1-r) \\
(1-l) x r & F(v) x
\end{array}\right] .
$$

We call $E(v), F(v), G(v)$ Peirce projections corresponding to $v$.

If $g$ is a linear functional on $A$ then, by abuse of notation, $E(v) g$ will denote $g \circ E(v)$, etc.

The following lemma is an easy consequence of the matrix representation.

LEMMA 2.1. For a partial isometry $v$ in a $C^{*}$-algebra $A$,

(a) $\|(E(v)+F(v)) x\|=\max \{\|E(v) x\|,\|F(v) x\|\} \leq\|x\|, x \in A$;

(b) $\|E(v) g\|+\|F(v) g\|=\|(E(v)+F(v)) g\| \leq\|g\|, g \in A^{*}$.

The next lemma puts an abstract $C^{*}$-algebra structure on the Peirce space $E(v) A$ of a partial isometry $v$. Its proof consists of straightforward calculations. For the third assertion, the following well-known result of Effros is needed: for a normal functional $f$ on a von Neumann algebra and a projection $e$ in the algebra, $f=f \cdot e \Leftrightarrow\|f\|=$ $\|f \cdot e\|$, where $f \cdot e$ is the functional $x \mapsto f(x e)$ (see [26, p. 140]). 
LEMMA 2.2. Let $v$ be a partial isometry in a $C^{*}$-algebra $A$.

1. $A_{v}:=v^{*} A r$, with $r=v^{*} v$, is a $C^{*}$-subalgebra of $A$, with unit $r$. If $A$ is a von Neumann algebra, so is $A_{v}$.

2. The map $x \mapsto v x$ is a linear isometric bijection of $A_{v}$ onto $E(v) A$ with inverse $a \mapsto v^{*} a$. Thus $E(v) A$ becomes a $C^{*}$-algebra with unit $v$ and operations

$$
a \cdot b:=a v^{*} b, \quad a^{\#}:=v a^{*} v .
$$

If $A$ is a von Neumann algebra, the adjoint of $x \mapsto v x$ is an isometry of $E(v) A_{*}$ onto $\left(A_{v}\right)_{*}$.

3. The map $\left.f \mapsto f\right|_{E(v) A}$ is an affine isometry of $\left\{f \in A^{*}: f(v)=\right.$ $\|f\|\}$ onto $(E(v) A)_{+}^{*}$. If $A$ is a von Neumann algebra, this map restricts to an affine isometry of $\left\{f \in A_{*}: f(v)=\|f\|\right\}$ onto $(E(v) A)_{*,+}$.

The Peirce space $E(v) A$ will occur frequently in the sequel. It will be denoted by $A_{2}(v)$. If $A$ is a von Neumann algebra, then by Lemma 2.2 , the normal state space of $A_{2}(v)$ is affinely isometric to the norm exposed face $F_{v}$ defined by $F_{v}=\left\{f \in A_{*}: f(v)=\|f\|=1\right\}$.

The next lemma examines the relation between partial isometries in a von Neumann algebra $A$ and norm exposed faces in the unit ball $A_{*, 1}$ of its predual. Note that if $u$ is a non-zero partial isometry in $A$, then by Lemma 2.2, $F_{u} \neq \varnothing$.

Partial isometries $u$ and $v$ are orthogonal if their left and right supports are orthogonal, i.e. $u u^{*} v v^{*}=0$ and $u^{*} u v^{*} v=0$. More generally, elements $x, y$ in a $C^{*}$-algebra are orthogonal if $x y^{*}=0=y^{*} x$.

LEMMA 2.3. For each $x$ in a von Neumann algebra $A$ with $\|x\|=1$ and $F_{x} \neq \varnothing$, there is a partial isometry $w \in A$ with $F_{x}=F_{w}$. Moreover, $x=y+w$ with $y$ orthogonal to $w$.

Proof. Let $|x|=\int_{0}^{1} \lambda d \lambda$ be the spectral decomposition of $|x|=$ $\left(x^{*} x\right)^{1 / 2}$. One can verify that $w:=u \int_{\{1\}} d \lambda$ satisfies the requirements of the lemma, where $x=u|x|$ is the polar decomposition of $x$. For details, see [7, Lemma 3].

Lemma 2.3 shows that the map $u \mapsto F_{u}$ from the set of partial isometries in a von Neumann algebra $A$ to the set of norm exposed faces in the unit ball $A_{*, 1}$ of the predual $A_{*}$ is onto. In fact, this map is also one-to-one. ${ }^{2}$ Indeed, by the Jordan decomposition of hermitian functionals on a von Neumann algebra and Lemma 2.2, for any partial isometry $u, E(u)^{*} A_{*}=\operatorname{sp} F_{u}$. Also, $u \in E(u) A$. Therefore,

\footnotetext{
${ }^{2}$ This will follow from [15, Proposition 1.6] once Theorem 2.11 is proved.
} 
$u$ is determined by its values on $\operatorname{sp} F_{u}$. It follows that if $F_{u}=F_{w}$, then $u=w$.

We next prove that the Peirce projections $E(v)$ and $F(v)$ corresponding to a partial isometry $v \in A$ are neutral. Although this follows from the result of Effros mentioned above, we prefer to give a direct proof of this fact. This proof introduces some tools which will be used later. In the first place, by defining a "triple product" $\{x y z\}:=\frac{1}{2}\left(x y^{*} z+z y^{*} x\right)$, and letting $A_{j}(v)$ denote the Peirce spaces corresponding to the partial isometry $v$, i.e.

$$
A_{2}(v)=E(v) A, \quad A_{1}(v)=G(v) A, \quad A_{0}(v)=F(v) A,
$$

we have by a simple matrix calculation,

$$
\left\{A_{i}(v), A_{j}(v), A_{k}(v)\right\} \subset A_{i-j+k}(v),
$$

where $A_{l}(v)=\{0\}$ if $l \notin\{0,1,2\}$. This notation will be justified by Theorem 2.11. Having introduced the triple product $\{x y z\}$ we also define an operator $D(x, y): A \rightarrow A$ by $D(x, y) z=\{x y z\}$ and the "cube" of $x$ as $x^{(3)}=D(x, x) x=\{x x x\}=x x^{*} x$. More generally, odd "powers" of elements of $A$ are defined inductively by $x^{(1)}=x$, $x^{(m)}=\left\{x x^{(m-2)} x\right\}$ for $m$ odd, $m \geq 3$. $D(x)$ will denote $D(x, x)$.

Note that $x$ and $y$ are orthogonal if and only if $D(x, y)=0$.

LEMMA 2.4. Let $v$ be a partial isometry in a $C^{*}$-algebra $A$ and let $\lambda$ be a complex number, $|\lambda|=1$. Define a linear map $S_{\lambda}=S_{\lambda}(v): A \rightarrow A$ by

$$
S_{\lambda}=S_{\lambda}(v)=\lambda^{2} E(v)+\lambda G(v)+F(v) .
$$

Then

(a) $S_{\lambda}$ preserves the triple product $\{x y z\}$, i.e.

$$
S_{\lambda}(\{x y z\})=\left\{S_{\lambda} x, S_{\lambda} y, S_{\lambda} z\right\} \quad \text { for } x, y, z \in A ;
$$

(b) $S_{\lambda}$ is an isometry of $A$ onto $A$.

Proof. (a) By the standard polarization formula

$$
\{a b c\}=\frac{1}{8} \sum_{\alpha^{4}=\beta^{2}=1} \alpha \beta(a+\alpha b+\beta c)^{(3)},
$$

it suffices to prove (a) in the special case $x=y=z$. Write $x=$ $\sum_{j=0}^{2} x_{j}$ with $x_{j} \in A_{j}(v)$. Then $S_{\lambda} x=\sum \lambda^{j} x_{j},\left\{S_{\lambda} x, S_{\lambda} x, S_{\lambda} x\right\}=$ 
$\sum_{i, j, k} \lambda^{i-j+k}\left\{x_{i} x_{j} x_{k}\right\}$, and $x^{(3)}=\sum_{i, j, k}\left\{x_{i} x_{j} x_{k}\right\}$. By (11), $\left\{x_{i} x_{j} x_{k}\right\} \in$ $A_{i-j+k}$. Therefore

$$
S_{\lambda}\left(x^{(3)}\right)=\sum_{i, j, k} \lambda^{i-j+k}\left\{x_{i} x_{j} x_{k}\right\}=\left(S_{\lambda} x\right)^{(3)} .
$$

(b)

$$
\begin{aligned}
\left\|S_{\lambda}(x)\right\|^{3} & =\left\|\left\{S_{\lambda}(x), S_{\lambda}(x), S_{\lambda}(x)\right\}\right\|=\left\|S_{\lambda}\left(x^{(3)}\right)\right\| \\
& \leq\left\|S_{\lambda}\right\|\left\|x^{(3)}\right\|=\left\|S_{\lambda}\right\|\|x\|^{3} .
\end{aligned}
$$

Thus $\left\|S_{\lambda}\right\| \leq 1$ and since $S_{\lambda} S_{\bar{\lambda}}=I, S_{\lambda}$ is isometric.

LEMMA 2.5. Let $v$ be a partial isometry in a $C^{*}$-algebra $A$, and let $x \in A_{2}(v) \cup A_{0}(v), y \in A_{1}(v), t \in \mathbf{C}$. Then for each positive integer $n$

$$
(x+t y)^{\left(3^{n}\right)}=x^{\left(3^{n}\right)}+t 2^{n} D\left(x^{\left(3^{n-1}\right)}\right) \cdots D\left(x^{(3)}\right) D(x) y+O\left(|t|^{2}\right) .
$$

Proof. By (11), $\{x y x\}=0$, and therefore

$$
\begin{aligned}
(x+t y)^{(3)} & =x^{(3)}+t\{x y x\}+2 t\{x x y\}+O\left(|t|^{2}\right) \\
& =x^{(3)}+2 t D(x) y+O\left(|t|^{2}\right) .
\end{aligned}
$$

The result now follows by induction: with

$$
x_{n}=x^{\left(3^{n}\right)} \text { and } y_{n}=2^{n} D\left(x_{n-1}\right) \cdots D(x) y,
$$

we have

$$
\begin{aligned}
(x+t y)^{\left(3^{n+1}\right)} & =\left(x_{n}+t y_{n}+O\left(|t|^{2}\right)\right)^{(3)}=\left(x_{n}+t y_{n}\right)^{(3)}+O\left(|t|^{2}\right) \\
& =x_{n}^{(3)}+2 t D\left(x_{n}\right) y_{n}+O\left(|t|^{2}\right) \\
& =x_{n+1}+t y_{n+1}+O\left(|t|^{2}\right) .
\end{aligned}
$$

The following proposition will imply the neutrality of the predual of a von Neumann algebra.

Proposition 2.6. Let $v$ be a partial isometry in a $C^{*}$-algebra $A$ and let $f \in A^{*}$.

(a) If $\|E(v) f\|=\|f\|$, then $E(v) f=f$;

(b) If $\|F(v) f\|=\|f\|$, then $F(v) f=f$.

Proof. (a) Since $\|E(v) f\|+\|F(v) f\|=\|E(v) f+F(v) f\| \leq\|f\|$, $F(v) f=0$. It remains to prove that $G(v) f=0$. Toward this end let $y \in G(v) A$. We are to prove that $f(y)=0$. We may assume $\|f\|=1$, 
$f(y) \geq 0$, and $\|y\| \leq 1$. For $\varepsilon>0$ choose $x \in E(v) A$ with $\|x\|=1$ and $f(x) \geq 1-\varepsilon$. Then for $t \in \mathbf{R}$,

$$
\|x+t y\| \geq f(x+t y)=f(x)+t f(y) \geq 1-\varepsilon+t f(y) .
$$

Therefore by Lemma 2.5

$$
\begin{aligned}
(1-\varepsilon+t f(y))^{3^{n}} & \leq\|x+t y\|^{3^{n}}=\left\|(x+t y)^{\left(3^{n}\right)}\right\| \\
& \leq\left\|x^{\left(3^{n}\right)}\right\|+t 2^{n}\|y\|+O\left(|t|^{2}\right)
\end{aligned}
$$

and so

$$
(1-\varepsilon)^{3^{n}}+3^{n} t f(y)(1-\varepsilon)^{3^{n}-1}+O\left(|t|^{2}\right) \leq 1+t 2^{n}\|y\|+O\left(|t|^{2}\right) .
$$

Letting $\varepsilon \rightarrow 0$, and dividing by $|t|$ results in

$$
f(y)+O(|t|) \leq\left(\frac{2}{3}\right)^{n}\|y\|+O(|t|) .
$$

Letting $t \rightarrow 0$, then $n \rightarrow \infty$ yields $f(y)=0$. The proof of (b) is similar.

The following gives a useful characterization of the relation $f \diamond g$.

LEMMA 2.7. Let $f$ and $g$ be normal functionals on a von Neumann algebra $A$. The following are equivalent.

(a) $f \diamond g$

(b) There exist orthogonal partial isometries $p, q$ in $A$ such that $f(p)=\|f\|$ and $g(q)=\|g\|$;

(c) There exist orthogonal partial isometries $p, q$ in $A$ such that $f=E(p) f$ and $g=E(q) g$.

Proof. (a) $\Rightarrow\left(\right.$ b): Suppose that $f \diamond g$. By the weak ${ }^{*}$-continuity of $f+g$ there is an $x \in A,\|x\|=1$ with $\langle f+g, x\rangle=\|f+g\|$. Choose a partial isometry $w$ by Lemma 2.3 with $F_{x}=F_{w}$. Then

$$
\|f\|+\|g\|=\|f+g\|=\langle f+g, w\rangle \leq|\langle f, w\rangle|+|\langle g, w\rangle| \leq\|f\|+\|g\| .
$$

Therefore $f(w)=\|f\|, g(w)=\|g\|$ so that $\varphi:=\left.f\right|_{A_{2}(w)}, \psi:=\left.g\right|_{A_{2}(w)}$ are positive normal functionals on the von Neumann algebra $A_{2}(w)$ with $\|\varphi-\psi\|=\|\varphi\|+\|\psi\|$. If $p$ and $q$ denote the support projections in $A_{2}(w)$ of $\varphi$ and $\psi$ respectively, then, as is well known, $p$ and $q$ are orthogonal projections in $A_{2}(w)$, i.e.

$$
\begin{gathered}
p=p \cdot p=p w^{*} p, \quad q=q \cdot q=q w^{*} q \\
p=p^{\#}=w p^{*} w, \quad q=q^{\#}=w q^{*} w \\
p \cdot q=p w^{*} q=0 .
\end{gathered}
$$


From these equations we have

$$
p=p \cdot p^{\#} \cdot p=p w^{*}\left(w p^{*} w\right) w^{*} p=p\left(w w^{*} p w^{*} w\right)^{*} p=p p^{*} p
$$

and

$$
p q^{*}=p\left(q^{\#}\right)^{*}=p\left(w q^{*} w\right)^{*}=p w^{*} q w^{*}=0 .
$$

Therefore $p$ and $q$ are orthogonal partial isometries in $A$.

We have $f(p)=\varphi(p)=\|\varphi\|=\|f\|$ and similarly, $g(q)=\|g\|$.

(b) $\Rightarrow$ (c): This follows from Proposition 2.6.

(c) $\Rightarrow(\mathrm{a})$ : If there exist orthogonal partial isometries $p, q$ in $A$ such that $f=E(p) f$ and $g=E(q) g$, then since $E(q)=F(p) E(q)$, it follows from Lemma 2.1 that $f \diamond g$.

We are now ready to show that the predual of a von Neumann algebra is WFS. Let $F_{x}$ be a norm exposed face in $A_{*, 1}$. With a partial isometry $w$ given by Lemma 2.3 such that $F_{x}=F_{w}$, let $S$ be the "Peirce reflection" with respect to $w$, i.e.

$$
S=E(w)-G(w)+F(w) .
$$

Since the fixed point set of $S$ is $E(w) A_{*} \oplus F(w) A_{*}$, the fact that $A_{*}$ is WFS will follow from the following two lemmas.

LEMMA 2.8. With $S$ as above,

(1) $S^{2}=I$;

(2) $S$ is an isometry of $A_{*}$ onto itself;

(3) $\operatorname{sp} F_{w}=E(w) A_{*}\left(=\left\{g \in A_{*}: E(w) g=g\right\}\right)$.

Proof. (1) is obvious and (2) follows from Lemma 2.4 with $\lambda=-1$. To prove (3), take $g \in F_{w}$. Then

$$
1=g(w)=\langle E(w) g, w\rangle \leq\|E(w) g\| \leq\|g\|=1,
$$

so that by Proposition 2.6, $g=E(w) g$. Therefore $F_{w} \subset E(w) A_{*}$ and

$$
\operatorname{sp} F_{w} \subset E(w) A_{*} \text {. }
$$

Since $F_{w}$ is the normal state space of the von Neumann algebra $A_{2}(w)$ and $E(w) A_{*}$ is its predual, (3) now follows from the Jordan and Cartesian decompositions of normal functionals.

We now have the following consequence of Lemmas 2.8 and 2.4. 
Corollary 2.9. Let $u$ be a partial isometry in a von Neumann algebra $A$ and suppose that $F_{u} \subset F_{x}$ for some $x \in A$ with $\|x\|=1$. Then $E(u) x=u$ and $G(u) x=0$.

Proof. Let

$$
x=x_{2}+x_{1}+x_{0}
$$

be the Peirce decomposition of $x$ with respect to $u$, i.e. $x_{2}=E(u) x$, $x_{1}=G(u) x, x_{0}=F(u) x$.

Since $F_{u} \subset F_{x}$,

$$
\left\langle F_{u}, x_{2}\right\rangle=\left\langle F_{u}, x\right\rangle=1=\left\langle F_{u}, u\right\rangle .
$$

On the other hand, $u$ and $x_{2}$ both vanish on $G(u)\left(A_{*}\right) \oplus F(u)\left(A_{*}\right)$. Thus $x_{2}=u$.

Let $y=u-i x_{1}-x_{0}$. Then $y=-S_{i}(u) x$ so that $\|y\|=1$ by Lemma 2.4. Therefore

$$
z:=\frac{1}{2}(x+y)=u+\mu x_{1} \quad \text { with } \mu=\frac{1}{2}(1-i)
$$

and $\|z\| \leq 1$. We now have $\|\{z z z\}\|=\|z\|^{3} \leq 1$, and therefore

$$
\left\|u+2|\mu|^{2}\left\{x_{1} x_{1} u\right\}\right\|=\left\|P_{2}(u)\{z z z\}\right\| \leq\|\{z z z\}\| \leq 1 .
$$

Since $\left\{x_{1} x_{1} u\right\}$ is a positive element of $A_{2}(u)$, it must be zero, and then it follows by a simple matrix calculation that $x_{1}=0$.

LEMMA 2.10. For any partial isometry $w$ in a von Neumann algebra $A$

$$
F_{w}^{\diamond}=F(w) A_{*} \quad\left(=\left\{g \in A_{*}: F(w) g=g\right\}\right)
$$

Proof. Let $g \in F(w) A_{*}, f \in F_{w}$. Then since $f \in E(w) A_{*}$, we have, by Lemma $2.1,\|f \pm g\|=\|f\|+\|g\|$, proving that $F_{w}^{\diamond} \supset F(w) A_{*}$.

Before proving the converse inclusion, note that since $A_{2}(w)$ is a von Neumann algebra with unit $w$,

$$
w=\sup \left\{e_{\varphi}: \varphi \in A_{2}(w)_{*,+}\right\},
$$

where $e_{\varphi}$ is the support projection in $A_{2}(w)$ of $\varphi$. By Zorn's lemma, there is an orthogonal family $\left\{e_{\alpha}\right\}$ such that

$$
w=\sum e_{\alpha}
$$

where $e_{\alpha}$ is the support of a normal functional $\varphi_{\alpha}$ on $A_{2}(w)$. As in the proof of Lemma 2.7, $\left\{e_{\alpha}\right\}$ is an orthogonal family of partial isometries in $A$. 
Now let $g \in F_{w}^{\diamond}$. We must show that $g \in F(w) A_{*}$. Consider $f_{\alpha}:=\varphi_{\alpha} \circ E(w) \in E(w) A_{*}$. Then $f_{\alpha}(w)=\varphi_{\alpha}(w)=\left\|\varphi_{\alpha}\right\|=\left\|f_{\alpha}\right\|$ so that $\left\|f_{\alpha}\right\|^{-1} f_{\alpha} \in F_{w}$ and therefore $f_{\alpha} \diamond g$ for all $\alpha$. By Lemma 2.7, there exist orthogonal partial isometries $p_{\alpha}, q_{\alpha}$ such that $f_{\alpha}\left(p_{\alpha}\right)=\left\|f_{\alpha}\right\|$, $g\left(q_{\alpha}\right)=\|g\|$ and $g=E\left(q_{\alpha}\right) g$. We now have

$$
\left\|f_{\alpha}\right\|^{-1} f_{\alpha} \in F_{w} \cap F_{p_{\alpha}}=F_{w_{\alpha}},
$$

for some partial isometry $w_{\alpha}$ (by Lemma 2.3). By Corollary 2.9, since $F_{w_{r}} \subset F_{p_{r}}$,

$$
p_{\alpha}=w_{\alpha}+b_{\alpha}
$$

with $b_{\alpha}$ orthogonal to $w_{\alpha}$. Therefore $w_{\alpha}$ is orthogonal to $q_{\alpha}$. Again by Corollary 2.9

$$
w=w_{\alpha}+c_{\alpha}
$$

with $c_{\alpha}$ orthogonal to $w_{\alpha}$. By direct calculation then, $w_{\alpha} \in A_{2}(w)$ and

$$
w_{\alpha}^{\#}=w_{\alpha} \cdot w_{\alpha}=w_{\alpha}
$$

Therefore $w_{\alpha}$ is a projection in $A_{2}(w)$ with $\varphi_{\alpha}\left(w_{\alpha}\right)=\left\|\varphi_{\alpha}\right\|$. Since $e_{\alpha}$ is the support projection of $\varphi_{\alpha}$, we have $e_{\alpha} \leq w_{\alpha}$ as projections in $A_{2}(w)$. Thus $e_{\alpha} \leq w_{\alpha}$ as partial isometries in $A$ and so $e_{\alpha}$ and $q_{\alpha}$ are orthogonal for all $\alpha$.

For the rest of this proof, it will be convenient to use the notation $l(u)=u u^{*}$ and $r(u)=u^{*} u$ for a partial isometry $u$.

By (12)

$$
l(w)=\sum l\left(e_{\alpha}\right) \quad \text { and } \quad r(w)=\sum r\left(e_{\alpha}\right)
$$

Therefore, for $x \in A$ and all $\alpha$, since $g=E\left(q_{\alpha}\right) g$

$$
g\left(l(w) x(1-r(w))=\lim \sum g\left(l\left(q_{\alpha}\right) l\left(e_{\alpha}\right) x(1-r(w))\right)=0,\right.
$$

and similarly

$$
g((1-l(w) x r(w))=0 \text { and } g(l(w) \operatorname{xr}(w))=0,
$$

i.e. $G(w) g=0$. Similarly, $E(w) g=0$.

THEOREM 2.11. The predual of a von Neumann algebra $A$ is a neutral strongly facially symmetric space in which the set of generalized tripotents coincides with the set of non-zero partial isometries. If $u$ is a partial isometry, then the generalized Peirce projections defined by $u$ agree with the Peirce projections defined by $u$, i.e.

$$
P_{2}(u)=E(u), \quad P_{1}(u)=G(u) \quad \text { and } \quad P_{0}(u)=F(u) .
$$


Proof. It follows from Lemmas 2.8 and 2.10 that $A_{*}$ is a WFS space.

Let $w$ be a non-zero partial isometry in $A$. Then $\|w\|=1$ and $F_{w} \neq$ $\varnothing$. Since $F_{w}^{\diamond}=F(w) A_{*}$, and $w \in A_{2}(w),\left\langle w, F_{w}^{\diamond}\right\rangle=\left\langle w, F(w) A_{*}\right\rangle=0$. Thus $w$ is a projective unit. But clearly, $S_{w}^{*} w=w$ so that $w$ is a generalized tripotent. Conversely let $u$ be a generalized tripotent. By Lemma 2.3 there is a partial isometry $w$ with $F_{u}=F_{w}$ and $y:=u-w$ orthogonal to $w$. We show that $y=0$ by showing that $g(y)=0$ for all $g \in A_{*}$. Since $y \in A_{0}(w)$ we may assume that $g \in F(w) A_{*}$ $\left(=F_{w}^{\diamond}=F_{u}^{\diamond}\right)$, and hence $g(w)=0$. Since $\left\langle u, F_{u}^{\diamond}\right\rangle=0, g(u)=0$. Therefore $g(y)=0$. Thus, the set of non-zero partial isometries coincides with the set of generalized tripotents.

It follows from Proposition 2.6 that $A_{*}$ is neutral.

By [15, Theorem 2.4], (13) holds.

To prove SFS let $F_{u} \subset F_{x}$ for some generalized tripotent $u$ and some element $x$ in $A$. Let

$$
x=x_{2}+x_{1}+x_{0}
$$

be the generalized Peirce decomposition of $x$ with respect to $u$, i.e. $x_{j}=P_{j}(u) x$ for $j=0,1,2$. We shall show that $x_{2}=u$ and then that $x_{1}=0$, which implies $S_{u}^{*} x=x$, as required for SFS.

Since $x_{2}=P_{2}(u)^{*} x$ and $F_{u} \subset F_{x}$,

$$
\left\langle F_{u}, x_{2}\right\rangle=\left\langle F_{u}, x\right\rangle=1=\left\langle F_{u}, u\right\rangle .
$$

On the other hand, since also $u=P_{2}(u)^{*} u, u$ and $x_{2}$ both vanish on $P_{1}(u)\left(A_{*}\right) \oplus P_{0}(u)\left(A_{*}\right)$. Thus $x_{2}=u$.

The proof that $x_{1}=0$ is identical to the last paragraph in the proof of Corollary 2.9 .

We can now combine Theorems 1.3 and 2.11 to obtain a geometric proof of the Tomita-Sakai-Effros polar decomposition of a normal functional. We state the version of this theorem from the book of Takesaki ([26, p. 140]).

THEOREM 2.12. Let $A$ be a von Neumann algebra, $f$ an element of the predual.

(1) There exists a pair $(\varphi, v)$ with the following properties:

(a) $\varphi$ is a positive normal functional on $A$, and $\|\varphi\|=\|f\|$;

(b) $v$ is a partial isometry of $A$ with $v^{*} v=\operatorname{supp} \varphi(:=$ the support of $\varphi)$;

(c) $f(x)=\varphi\left(v^{*} x\right), \varphi(x)=f(v x)$, for $x \in A$. 
(2) Let $\psi$ be a positive normal functional on $A, u$ a partial isometry of $A$ with $u^{*} u=\operatorname{supp} \psi$ and $f(x)=\psi\left(u^{*} x\right)$, for $x \in A$. Then $\psi=\varphi$, $u=v$.

Proof. For notation's sake, assume $\|f\|=1$.

(1) Given $f$, choose a generalized tripotent $v$ satisfying the conditions in Theorem 1.3. Define $\varphi \in A_{*}$ by the rule $\varphi(x)=f(v x)$, for $x \in A$. Then $\|\varphi\| \leq\|f\|$ and $\varphi\left(v^{*} v\right)=f(v)=\|f\|$ so that $\|\varphi\|=\|f\|$. Also, $v$ is a partial isometry and, with $r=v^{*} v$, we have $\varphi(1)=\varphi(r+(1-r))=\varphi(r)=1$ so that $\varphi \in A_{*,+}$ and $\operatorname{supp} \varphi \leq r$. If $p$ is a projection in $A$ with $p \leq r$, then $v p \leq v$ by Lemma 2.2. Hence by (d) of Theorem 1.3, $\varphi(p)=f(v p)>0$, proving that $r=\operatorname{supp} \varphi$. Finally, $\varphi\left(v^{*} x\right)=f\left(v v^{*} x\right)=f(x)$ for $x \in A$, by neutrality.

(2) Obviously, $\|f\| \leq\|\psi\|$. On the other hand, $\|\psi\|=\psi\left(u^{*} u\right)=$ $f(u)$ so $\|f\|=\|\psi\|$. Since $u$ is a partial isometry, it is a generalized tripotent with $f(u)=\psi\left(u^{*} u\right)=1$, i.e. $f \in F_{u}$. Using the isomorphism of Lemma 2.2, we see that $\psi$ on $A_{u}$ corresponds to $f$ on $E(u) A$ and since $\psi$ is faithful on $A_{u}, f$ is faithful on $E(u) A$.

By [14, Corollary 1.3b], if $g \in\{f\}^{\diamond},\|g\|=1$, there exist orthogonal norm exposed faces $F_{x}, F_{y}$ with $f \in F_{x}, g \in F_{y}$. Thus $f \in F_{x} \cap F_{u}=$ $F_{w}$ for some projection $w$ in $E(u) A$. By the faithfulness of $f$ on $E(u) A, w=u$ and therefore $F_{u} \subset F_{x}$, and $F_{u}^{\diamond} \supset F_{x}^{\diamond} \supset F_{y}$. Since $\left\langle u, F_{u}^{\diamond}\right\rangle=0$ and $g \in F_{y}, g(u)=0$. Therefore $\left\langle u,\{f\}^{\diamond}\right\rangle=0$. By the uniqueness part of Theorem 1.3, $u=v$ and $\psi(x)=f(u x)=f(v x)=$ $\varphi(x)$.

3. Application to $J B^{*}$-triples. A $J B^{*}$-triple (or Banach Jordan Triple System) is a complex Banach space with a certain ternary algebraic structure arising in the study of bounded symmetric domains in finite and infinite dimensions ([21]). The class includes $C^{*}$-algebras and $J B^{*}$-algebras as well as the range of an arbitrary contractive projection on a $C^{*}$-algebra ([11]). A $J B W^{*}$-triple is a $J B^{*}$-triple which is the dual of a Banach space.

Recent results in the analytic and algebraic theory of Jordan triple systems have led to a good understanding of their structure. The algebraic structure of a $J B^{*}$-triple is uniquely determined by the isometric structure of its unit ball. It is also uniquely determined by the holomorphic structure of its unit ball. Hence, results on the geometry of $J B^{*}$-triples underlie much of this structure theory. Some of the principal advances in this direction include the following. 


\section{Geometric Structure}

(1) Affine geometric structure of a $J B W^{*}$-triple and its predual: facial structure ([10]), and state space properties ([12]).

(2) Geometric tools: stability under contractive projections ([22], [25]), and weak ${ }^{*}$ continuity of the triple product ([3],[8],[16]).

(3) Foundations in holomorphy ([20], [21], [27], [30]).

\section{Algebraic Structure}

(4) Gelfand-Naimark Theorem ([13]).

(5) Classification Theory for $J B W^{*}$-triples: atomic ([6], [24]), type I ([17]), and continuous ([18]).

This theory has had and continues to have significant connections to other areas of analysis. (An introductory survey of several applications of Jordan theory to analysis, operator theory, and the foundations of quantum mechanics has been given by Upmeier [29].) A Riemann Mapping Theorem for the bounded symmetric domains in complex Banach spaces was proven by Kaup ([21]), who showed that there is a one-to-one correspondence between the biholomorphic equivalence classes of these domains and the algebraic isomorphism classes of $J B^{*}$-triples. Upmeier has developed a definitive theory of multivariable Toeplitz operators over finite dimensional bounded symmetric domains using the correspondence between these domains and $J B^{*}$-triples (cf. [29, Lecture 8]).

Since the analog of the polar decomposition is known in the context of $J B W^{*}$-triples, and since the latter are intrinsically geometric objects, it should not be surprising that this polar decomposition is a consequence of the theory of facially symmetric spaces. Indeed, we have the following two theorems, which are analogues of the main results of $\S 2$. In order to state these results we now recall the definitions and basic properties of $J B^{*}$-triples (cf. [28]).

A $J B^{*}$-triple is a complex Banach space $U$ endowed with a continuous sesqui-linear map $D: U \times U \rightarrow \mathscr{B}(U)$, such that for $x \in U$, $D(x, x)$ is Hermitian positive, $\|D(x, x)\|=\|x\|^{2}$, and, setting $\{x y z\}:=$ $D(x, y) z$, one has

$$
\{x y z\}=\{z y x\}
$$

and

$$
\{x y\{u v z\}\}+\{u\{y x v\} z\}=\{\{x y u\} v z\}+\{u v\{x y z\}\} .
$$

For example, a $C^{*}$-algebra is a $J B^{*}$-triple with

$$
\{x y z\}:=\frac{1}{2}\left(x y^{*} z+z y^{*} x\right)
$$


and a $J B^{*}$-algebra is a $J B^{*}$-triple with

$$
\{x y z\}:=\left(x \circ y^{*}\right) \circ z+\left(z \circ y^{*}\right) \circ x-(z \circ x) \circ y^{*},
$$

where $\circ$ denotes the Jordan product in the $J B^{*}$-algebra.

Denote by $Q$ the quadratic operator on the $J B^{*}$-triple $U$, i.e. $Q(x) y$ $=\{x y x\}$, for $x, y \in U$. Then set

$$
Q(x, z)=\frac{1}{2}(Q(x+z)-Q(x)-Q(z))
$$

so that $Q(x, z) y=\{x y z\}$, for $x, y, z \in U$.

A tripotent in a $J B^{*}$-triple is an element $e$ with $e=\{e e e\}$. Define the Peirce projections $P_{k}(e), k=0,1,2$ relative to a tripotent $e$ by

$$
\begin{gathered}
P_{2}(e)=Q(e)^{2}, \quad P_{1}(e)=2\left(D(e, e)-Q(e)^{2}\right) \\
P_{0}(e)=I-2 D(e, e)+Q(e)^{2} .
\end{gathered}
$$

Note that $\sum P_{k}(e)=I$ and $D(e, e)=P_{2}(e)+\frac{1}{2} P_{1}(e)$. It follows from purely algebraic considerations that each $P_{j}(e)$ is idempotent and that $P_{k}(e) P_{j}(e)=0$ if $k \neq j$. Let $U_{k}(e)$ be the range of $P_{k}(e)$. The Peirce decomposition is

$$
U=U_{2}(e) \oplus U_{i}(e) \oplus U_{0}(e)
$$

and $U_{k}(e)$ is the $k / 2$-eigenspace of $D(e, e)$. We have the following fundamental properties:

$$
\begin{gathered}
\left\{U_{i}(e) U_{j}(e) U_{k}(e)\right\} \subset U_{i-j+k}(e) ; \\
\left\{U_{2}(e) U_{0}(e) U\right\}=\left\{U_{0}(e) U_{2}(e) U\right\}=0
\end{gathered}
$$

The Peirce 2-space $U_{2}(e)$ is a complex Jordan *-algebra, with product $x \circ y=\{x e y\}$, unit $e$, and involution $z^{\#}=\{e z e\}$. If $U$ is a $J B^{*}$-triple, then $U_{2}(e)$ is a $J B^{*}$-algebra with these operations.

THEOREM 3.1. The predual $U_{*}$ of a $J B W^{*}$-triple $U$ is a neutral strongly facially symmetric space in which the set of generalized tripotents coincides with the set of tripotents. If $u$ is a generalized tripotent, then the generalized Peirce projections associated with $u$ agree with the adjoints of the Peirce projections associated with $u$.

Proof. If $F_{x}$ is a norm exposed face in $U_{*, 1}$ then by [12, Proposition 8], there is a tripotent $u$ in $U$ with $F_{x}=F_{u}$. By [12, Lemma 1.1], $S_{-1}(u):=P_{2}(u)-P_{1}(u)+P_{0}(u)$ is an isometric symmetry. By the 
Jordan decomposition of hermitian normal functionals on a $J B W^{*}$ algebra, $P_{2}(u) U_{*}=\operatorname{sp} F_{u}$. By an algebraic analog of Lemma 2.7 one can show that $P_{0}(u) U_{*}=F_{u}^{\diamond}$. Therefore $U_{*}$ is $W F S$.

By an argument similar to that in Theorem 2.11 one can show that the set of generalized tripotents coincides with the set of non-zero tripotents.

By [12, Proposition 1], $U_{*}$ is neutral.

By [15, Theorem 2.4], the generalized Peirce projections agree with the adjoints of the Peirce projections. Now by [12, Lemma 1.6] and the argument of Theorem 2.11 it follows that $U_{*}$ is SFS.

THEOREM 3.2 (Proposition 2 of [13]). For each functional $f$ in the predual of a $J B W^{*}$-triple, there is a unique tripotent $e$ such that $f=$ $P_{2}(e)^{*} f$ and $\left.f\right|_{U_{2}(e)}$ is a faithful normal positive functional on the $J B W^{*}$-algebra $U_{2}(e)$.

Proof. Since $U_{*}$ is a neutral strongly facially symmetric space, given $f$ (with $\|f\|=1$ ), there is a generalized tripotent $e$ satisfying the conditions in Theorem 1.3. Then $e$ is a tripotent and since $f \in F_{e}$, $\|f\|=\left\|P_{2}(e)^{*} f\right\|$ so by [12, Proposition 1], $f=P_{2}(e)^{*} f$. Since $\left.f\right|_{U_{2}(e)}(e)=1, f$ is positive, and since $f$ is normal, so is $\left.f\right|_{U_{2}(e)}$. Let $p$ be a projection in $U_{2}(e)$. Then $p$ is a tripotent in $U$ with $p \leq e$ and therefore $f(p)>0$ by (d) of Theorem 1.3. The existence is proved. Here we have used [15, Lemma 4.2].

To prove the uniqueness, it suffices, by Theorem 1.3 to show that any tripotent $u$ satisfying the above conditions also satisfies $f \in F_{u}$ and $\left\langle u,\{f\}^{\circ}\right\rangle=0$.

Since $f$ is a positive functional on the $J B^{*}$-algebra $U_{2}(u), f \in F_{u}$. Now continue as in the last paragraph of the proof of Theorem 2.12.

\section{REFERENCES}

[1] E. Alfsen, H. Hanche-Olsen, and F. Shultz, State spaces of $C^{*}$-algebras, Acta Math., 144 (1980), 267-305.

[2] E. Alfsen and F. Shultz, State spaces of Jordan algebras, Acta Math., 140 (1978), 155-190.

[3] T. J. Barton and R. M. Timoney, Weak* continuity of Jordan triple products and applications, Math. Scand., 59 (1986), 177-191.

[4] A. Connes, Caractérisation des espaces vectoriels ordonnés sous jacents aux algèbres de von Neumann, Ann. Inst. Fourier, Grenoble, 24 (1974), 121-155. 
[5] T. Dang, Real isometries of $J B^{*}$-triples, preprint, 1987.

[6] T. Dang and Y. Friedman, Classification of $J B W^{*}$-triple factors and applications, Math. Scand., 61 (1987), 292-330.

[7] T. Dang, Y. Friedman, and B. Russo, Affine geometric proofs of the BanachStone theorems of Kadison and Kaup, Proc. of the 1987 Great Plains Operator Theory Seminar, Rocky Mountain J. Math., (to appear).

[8] S. Dineen, Complete holomorphic vector fields on the second dual of a Banach space, Math. Scand., 59 (1986), 131-142.

[9] E. Effros, Order ideals in a $C^{*}$-algebra and its dual, Duke Math. J., 30 (1963), 391-412.

[10] C. M. Edwards and G. T. Rüttiman, On the facial structure of the unit balls in a $J B W^{*}$-triple and its predual, preprint, 1987.

[11] Y. Friedman and B. Russo, Solution of the contractive projection problem, J. Funct. Anal., 60 (1985), 56-79.

[12] - Structure of the predual of a $J B W^{*}$-triple, J. Reine Angew. Math., 356 (1985), 67-89.

[13] , A Gelfand-Naimark Theorem for JB*-triples, Duke Math. J., 53 (1986), 139-148.

[14] A geometric spectral theorem, Quart. J. Math. Oxford (2), 37 (1986), 263-277.

[15] Affine structure of facially symmetric spaces, Math. Proc. Camb. Philos. Soc. (to appear).

[16] G. Horn, Characterization of the predual and ideal structure of a $\mathrm{JBW}^{*}$-triple, Math. Scand., 61 (1987), 117-133.

[17] Classification of JBW* -triples of type I, Math. Z., 196 (1987), 271-291.

[18] G. Horn and E. Neher, Classification of continuous $J B W^{*}$-triples, Trans. Amer. Math. Soc., 306 (1988), 553-578.

[19] B. Iochum, Cônes Autopolaires et Algèbres de Jordan, Lecture Notes in Math. 1049, Springer-Verlag, New York, 1984.

[20] W. Kaup, Algebraic characterization of symmetric complex Banach manifolds, Math. Ann., 228 (1977), 39-64.

[21] _ A Riemann Mapping Theorem for bounded symmetric domains in complex Banach spaces, Math. Z., 183 (1983), 503-529.

[22] Contractive projections on Jordan $C^{*}$-algebras and generalizations, Math. Scand., 54 (1984), 95-100.

[23] K. McCrimmon, Compatible Peirce decompositions of Jordan triple systems, Pacific J. Math., 103 (1982), 57-102.

[24] E. Neher, Jordan Triple Systems by the Grid Approach, Lecture Notes in Math., 1280, Springer-Verlag, New York 1987.

[25] L. Stachó, A projection principle concerning biholomorphic automorphisms, Acta Sci. Math., 44 (1982), 99-124.

[26] M. Takesaki, Theory of Operator Algebras I, Springer-Verlag, New York, 1979.

[27] H. Upmeier, Über die Automorphismengruppen von Banach Mannigfaltigkeiten mit Invarianter Metrik, Math. Ann., 223 (1976), 279-288.

[28] _ Symmetric Banach Manifolds and Jordan $C^{*}$-algebras, North-Holland, 1985.

[29] _ Jordan Algebras in Analysis, Operator Theory, and Quantum Mechanics, AMS-CBMS Regional Conference Series no. 67, 1987. 
[30] J. P. Vigué, Le groupe des automorphismes analytiques d'un domaine borné d'un espace de Banach complexe. Application aux domains bornés symmétriques, Ann. Sci. Ec. Norm. Sup., $4^{e}$ serie, 9 (1976), 203-282.

Received March 10, 1988. Supported by NSF Grant No. DMS 8603064.

Jerusalem College of Technology

P.O. BOX 16031

JERUSALEM, ISRAEL $91-160$

AND

UNIVERSITY OF CALIFORNIA

IRVINE, CA 92717 


\section{PACIFIC JOURNAL OF MATHEMATICS EDITORS}

\author{
V. S. VARADARAJAN \\ (Managing Editor) \\ University of California \\ Los Angeles, CA 90024 \\ Herbert Clemens \\ University of Utah \\ Salt Lake City, UT 84112 \\ THOMAS ENRIGHT \\ University of California, San Diego \\ La Jolla, CA 92093
}

R. FINN

Stanford University

Stanford, CA 94305

HeRmanN FlaschKa

University of Arizona

Tucson, AZ 85721

VAUGHAN F. R. JONES

University of California

Berkeley, CA 94720

STEVEN KeRCKHOFF

Stanford University

Stanford, CA 94305
RoBION KIRBY

University of California

Berkeley, CA 94720

C. C. MOore

University of California

Berkeley, CA 94720

HAROLD STARK

University of California, San La Jolla, CA 92093

\section{ASSOCIATE EDITORS}
R. ARENS
E. F. BECKENBACH
B. H. NeUmanN
F. WolF
K. YosHIDA (1906-1982)

\section{SUPPORTING INSTITUTIONS}
UNIVERSITY OF ARIZONA
UNIVERSITY OF BRITISH COLUMBIA CALIFORNIA INSTITUTE OF TECHNOLOGY UNIVERSITY OF CALIFORNIA MONTANA STATE UNIVERSITY UNIVERSITY OF NEVADA, RENO NEW MEXICO STATE UNIVERSITY OREGON STATE UNIVERSITY
UNIVERSITY OF OREGON UNIVERSITY OF SOUTHERN CALIFORNIA STANFORD UNIVERSITY UNIVERSITY OF HAWAII UNIVERSITY OF TOKYO UNIVERSITY OF UTAH WASHINGTON STATE UNIVERSITY UNIVERSITY OF WASHINGTON 


\section{Pacific Journal of Mathematics}

\section{Vol. 137, No. $1 \quad$ January, 1989}

V. S. Varadarajan, Henry Abel Dye $\ldots \ldots \ldots \ldots \ldots \ldots \ldots \ldots \ldots \ldots$ iii

Huzihiro Araki, An application of Dye's theorem on projection lattices to orthogonally decomposable isomorphisms $\ldots \ldots \ldots \ldots \ldots \ldots \ldots \ldots \ldots$

Richard Arens, The limit of a sequence of squares in an algebra need not be

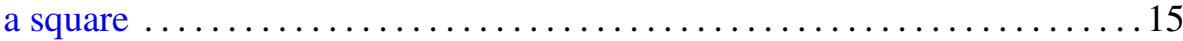

William Arveson, An addition formula for the index of semigroups of

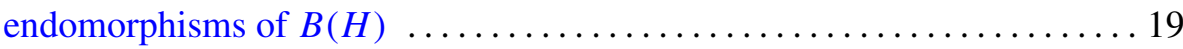

Robert James Blattner and Susan Montgomery, Crossed products and

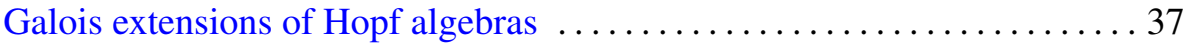

Erik Christensen and Allan M. Sinclair, On the vanishing of $H^{n}\left(\mathscr{A}, \mathscr{A}^{*}\right)$

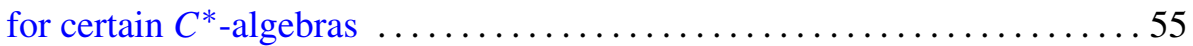

Philip C. Curtis, Jr. and Michael M. Neumann, Nonanalytic functional

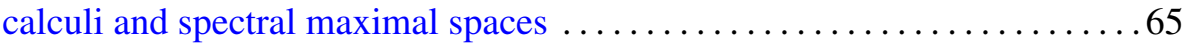

George A. Elliott and David E. Handelman, Addition of $C^{*}$-algebra extensions

Yaakov Friedman and Bernard Russo, Some affine geometric aspects of

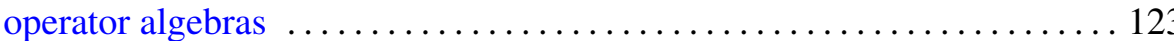

Valentin Ya. Golodets and Sergey D. Sinelshchikov, Regularization of actions of groups and groupoids on measured equivalence relations . . . 145

Irving Kaplansky, CCR-rings 155

Hideki Kosaki, Characterization of crossed product (properly infinite case)

Gert Kjærgaard Pedersen, Three quavers on unitary elements in $C^{*}$-algebras

Sorin Popa, Relative dimension, towers of projections and commuting squares of subfactors

Martin E. Walter, On a new method for defining the norm of

Fourier-Stieltjes algebras 